\title{
Neutrophil-to-lymphocyte ratio and pulse wave velocity in patients with controlled systemic hypertension - a preliminary report
}

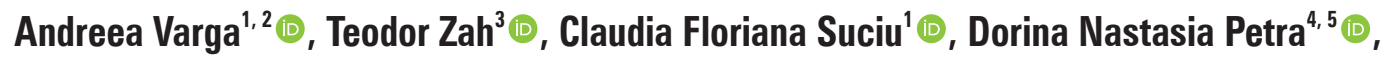 \\ Corneliu Florin Buicu ${ }^{6}$ (1) \\ ${ }^{1}$ Family Medicine, Department ME2 — Clinical Disciplines, Faculty of Medicine in English, George Emil Palade University of Medicine, \\ Pharmacy, Science and Technology of Targu Mures, Romania \\ ${ }^{2}$ Internal Medicine II Clinic — Cardiology Department, Emergency Clinical County Hospital Targu Mures, Romania \\ ${ }^{3}$ The Oncology Institute "Prof. Dr. Ion Chiricuta", Cluj-Napoca, Romania \\ ${ }^{4}$ Family Medicine, Department M4, Faculty of Medicine, George Emil Palade University of Medicine, Pharmacy, \\ Science and Technology of Targu Mures, Romania \\ ${ }^{5}$ Internal Medicine II Clinic, Emergency Clinical County Hospital, Targu Mures, Romania \\ ${ }^{6}$ Public Health and Management, Department M2 - Functional and Complementary Sciences, Faculty of Medicine, \\ George Emil Palade University of Medicine, Pharmacy, Science and Technology of Targu Mures, Romania
}

\begin{abstract}
Background. Increased arterial stiffness assessed by pulse wave velocity (PWV) measurement is a marker of arterial wall dysfunction and has an independent predictive value for adverse cardiovascular outcomes. A positive correlation between the neutrophil-to-lymphocyte ratio (NLR) and PWV has been reported in chronic inflammatory conditions and the general population as well.

Furthermore, an association between NLR and PWV has been assumed in hypertensive patients. However, the available data are scarce.

The objective of the study was to validate the association between NLR and PWV in a homogenous group of controlled-hypertensive patients without chronic inflammatory conditions.

Material and methods. A retrospective observational study was conducted in outpatient cardiology and a general practice. A total number of 25 already on-target treated essential hypertensive, non-diabetic and non-chronic kidney disease (non-CKD) patients were selected. PWV was automatically calculated for each patient using the ABPM BPLab device. The following laboratory data were collected: complete blood count, fibrinogen, alkaline phosphatase, lactate dehydrogenase, uric acid, serum glucose, total cholesterol, triglycerides, iron, calcium, and creatinine. Neutrophil-to-lymphocyte ratio was calculated. Antihypertensive treatment classes were also assessed.

Results. A correlation between increased NLR and PWV in a homogenous group of controlled-hypertensive patients was identified.

Conclusions. There is an evident relation between increased NLR and increased PWV in controlled hypertensive patients without evidence of chronic inflammatory conditions.
\end{abstract}

Key words: neutrophil-to-lymphocyte ratio; pulse wave velocity; hypertension

Arterial Hypertens. 2020, vol. 24, no. 2, pages: 67-73

DOI: $10.5603 /$ AH.a2020.0008

Address for correspondence: Assistant Claudia Floriana SUCIU, MD, PhD candidate

Family Medicine, Department ME2 - Clinical Disciplines, Faculty of Medicine in English, George Emil Palade University of Medicine, Pharmacy, Science and Technology of Targu Mures, 35 Revolutiei st, 540042, Targu Mures, Romania, tel: (+40) 738315 470, fax: (+40) 265211011 ; e-mail: claudia.suciu@umfst.ro

VM Copyright $@ 2020$ Via Medica, ISSN 2449-6170 


\section{Introduction}

The leading cause of morbidity and mortality worldwide is represented by cardiovascular diseases (CVD); hypertension is considered a major risk factor for heart disease, being ranked the third most common cause leading to disability in the world [1].

Arterial stiffness is characterized by degeneration of extracellular matrix in the media layer of elastic arteries with loss of elastic fibres, and increased fibrosis as a consequence of increased cyclic stress [2]. The changes in arterial stiffening are morphologically different from atherosclerosis, a process that involves the intima layer and is characterized by patchy intimal thickening with subsequent lumen narrowing [3].

Increased arterial stiffness is considered to be the initial indicator of arterial wall dysfunction and has an independent predictive value for adverse CV outcomes, along with traditional CV risk factors [4-6]. Patients with diabetes mellitus, metabolic syndrome, or chronic inflammation status are at high risk of developing increased arterial stiffness $[7,8]$.

Pulse wave velocity (PWV) is the most commonly used method for arterial stiffness assessment [9].

As arterial stiffening affects mainly the proximal arteries, the stiffness gradient between proximal and distal arteries will subside leading to decreased wave reflection from the resistance vessels, and consequently increased pressure in the microcirculation, with negative effects on the perfused organs, particularly brain and kidney [10].

Additionally, a reduced arterial filling capacity as a consequence of increased stiffness leads to an enhanced pulse pressure (PP) and causes prematurely recoil of the reflected waves with a consequent enhancement of late systolic pressure and gradual left ventricular hypertrophy development $[11,12]$.

Although PWV is indisputably an excellent tool for CV risk stratification, it also bears relevant limitations [13]. Pulse wave velocity is influenced by many clinical factors like age, blood pressure (BP) and other traditional CV risk factors [14]. For proper evaluation, target BP control should be achieved before PWV determination [9].

As a result of the age-related reduction in nitric oxide synthesis and increased vasoconstrictor availability, elder individuals present impairment in vascular function leading to decreased resistance to oxidative stress and changes in vascular structure with wall thickening and increased stiffness in conduit arteries [15-17]. Consequently, the function of preserving a constant BP against a pulsating blood flow is more impaired in elderly individuals.
For PWV assessment, invasive and non-invasive methods are accessible, non-invasive methods being currently available in clinical practice. Arterial stiffness can be determined by non-invasive carotid-femoral PWV computation using a tonometry device $[14,15]$.

Pulse wave velocity cut-off values vary among different populations as a consequence of the many confounders. In middle-aged patients with hypertension, a PWV value higher than $10 \mathrm{~m} / \mathrm{s}$ is associated with significant aortic function deterioration $[15,18]$.

Ambulatory 24-hour PWV analysis is a validated arterial stiffness measurement that also involves assessment of central arterial pressure (CAP) and augmentation index (AIx) calculations, both being able to be estimated from the common carotid artery or a peripheral artery (i.e. brachial artery) waveform [19-21].

The neutrophil-to-lymphocyte ratio (NLR) is currently investigated as an inflammatory marker. NLR is associated with atherosclerosis, hypertension, coronary artery disease, acute coronary syndromes and other known inflammatory conditions; additionally, NLR may be an indicator of disease severity of coronary artery disease and calcific atherosclerosis [23-27].

A positive correlation between NLR and PWV in the general population has been reported in previous studies, but also in research focused on chronic inflammatory conditions [28-30]. Furthermore, an association between PWV and NLR in hypertensive patients identified NLR as a valid index for arterial stiffness [31]. Nevertheless, a relationship between NLR and arteriosclerosis in hypertensive patients is scarce.

The premise of our study was to validate the association between NLR and arterial stiffness assessed by PWV in a homogenous group of controlled-hypertensive patients.

\section{Material and methods}

A retrospective observational study was conducted in outpatient cardiology and a general practice. Data were collected after the study protocol approval by local Ethics Committee no. 1172/14/01/2019. All the subjects afforded written informed consent.

A total number of fifty-nine unselected, already treated at target essential hypertensive adult patients underwent a 24-hour ambulatory blood pressure monitoring (ABPM) evaluation from June 2016 to June 2017, using a BPLab device (BPLab GmbH, Schwalbach am Taunus, Germany); derived used parameters were: mean arterial pressure (MAP) and pulse wave velocity (PWV). 
Subjects with any of the following concomitant diagnosis: atrial fibrillation, frequent ectopic beats, secondary hypertension, documented or suspected cardiomyopathy, chronic inflammatory conditions, infectious diseases, or malignant tumours were excluded from statistical analysis. In addition, we did not include in our research patients with antihypertensive regimens containing amlodipine or valsartan. Patients were instructed to avoid alcohol, caffeinecontaining products and substantial meal 8 hours before and during 24-hour monitoring.

The 24-hour BP reports along with the laboratory tests performed within the monitoring time frame, anthropometric and demographic data were retrospectively collected from an integrated electronic database.

The carotid-femoral PWV was automatically calculated using the ABPM BPLab device, which is an automatic, validated device to measure the time delay between the rapid upstroke of the feet of simultaneously recorded pulse waves in the carotid and the femoral arteries, over a 24 hours period [32]. The "surrogate" length of the aorta was measured noninvasively with a non-elastic tape measure, between carotid and femoral artery. In order to reduce variations in the measurements, one trained technician performed PWV data collection.

With respect to the inclusion and exclusion criteria, a total number of 25 already treated at target essential hypertensive, non-diabetic and non-CKD patients were included in the study. Out of these patients, seventeen were males (68.0\%) and eight were females (32.0\%).

The following laboratory data were collected: complete blood count, fibrinogen, alkaline phos- phatase, lactate dehydrogenase, uric acid, serum glucose, total cholesterol, triglycerides, iron, calcium, and creatinine. Neutrophil-to-lymphocyte ratio was calculated. Glomerular filtration rate was estimated using the CKD-EPI formula. Antihypertensive treatment classes were also assessed.

The association between the qualitative variables was assessed using the Student's t-test. P values $<0.05$ were considered statistically significant, and all confidence intervals are $95 \%$. Linear regression analysis was performed in order to test the relationship between selected parameters, Pearson productmoment correlation coefficient $(r)$ was used to assess the strength of the association. The statistical analysis was performed using SPSS 22.0 (IBM Corp. Released 2013. IBM SPSS Statistics for Windows, Version 22.0. Armonk, USA), and Graph Pad Prism 8.1.2 (227) software (GraphPad Software Inc., San Diego, USA).

\section{Results}

The mean age of our study group (25 patients) was $54.96 \pm 13.03$ (range 29-87) years old; mean age in the male subgroup was $53.45 \pm 14.59$ years old and mean age in the female subgroup was $55.68 \pm 14.6$ years old, respectively. Subject's body mass index (BMI) was $29.77 \pm 4.10 \mathrm{~kg} / \mathrm{m}^{2}$ (range 20.50-35.50 $\mathrm{kg}$ ). Overweight was diagnosed in $45 \%$ of the study subjects, $45 \%$ have obesity grade I and 5\% obesity grade II. Distinctive laboratory descriptive data and their correlation to PWV are depicted in Table 1.

Table 1. Laboratory descriptive data and their correlation to pulse wave velocity (PWV)

\begin{tabular}{|c|c|c|c|c|c|c|}
\hline \multirow{2}{*}{ Parameters } & \multirow{2}{*}{$\mathbf{N}$} & \multirow{2}{*}{ Min } & \multirow{2}{*}{ Max } & \multirow{2}{*}{ Range } & \multicolumn{2}{|c|}{ PWV } \\
\hline & & & & & $\mathrm{p}$ value & Pearson's r \\
\hline Neutrophils (NV: $1.5-7.5 \times 10^{3} / \mu \mathrm{L}$ ) & 25 & 2.46 & 8.45 & 5.99 & \multicolumn{2}{|c|}{-} \\
\hline Lymphocytes (NV: $1.0-4.0 \times 10^{3} / \mu \mathrm{L}$ ) & 25 & 0.82 & 4.00 & 3.18 & \multicolumn{2}{|c|}{-} \\
\hline NLR & 25 & 9.10 & 15.70 & 6.60 & \multicolumn{2}{|c|}{-} \\
\hline Fibrinogen (NV: $1.5-4.0 \mathrm{~g} / \mathrm{L}$ ) & 12 & 2.40 & 5.50 & 3.10 & 0.04 & 0.58 \\
\hline Alkaline phosphatase (NV: 100-300 U/L) & 21 & 57.00 & 352.00 & 295.00 & 0.32 & 0.22 \\
\hline Lactate dehydrogenase (NV: $240-480 \mathrm{U} / \mathrm{L}$ ) & 18 & 149.00 & 440.00 & 291.00 & 0.97 & -0.01 \\
\hline Uric acid (NV: 200-400 U/L) & 23 & 173.30 & 749.00 & 575.70 & 0.11 & 0.33 \\
\hline Serum glucose (NV: 4-5.4 mmol/L) & 25 & 4.41 & 9.99 & 5.58 & 0.01 & 0.57 \\
\hline Total cholesterol (NV: $2.8-5.2 \mathrm{mmo} / \mathrm{L}$ ) & 25 & 3.15 & 8.48 & 5.33 & 0.97 & 0.01 \\
\hline Triglyceride (NV: 0.55-1.90 mmol/L) & 22 & 0.61 & 10.60 & 9.99 & 0.41 & 0.03 \\
\hline Iron (NV: $9.0-30.4 \mu \mathrm{mol} / \mathrm{L}$ ) & 15 & 9.20 & 23.30 & 14.10 & 0.63 & 0.13 \\
\hline Total calcium (NV: $2.15-2.57 \mathrm{mmo} / \mathrm{L}$ ) & 16 & 1.94 & 2.53 & 0.59 & 0.75 & 0.08 \\
\hline
\end{tabular}

$\mathrm{N}$ - number of patients; Min — minimum; Max - maximum; NV — normal values; NLR — neutrophil-to-lymphocyte ratio 


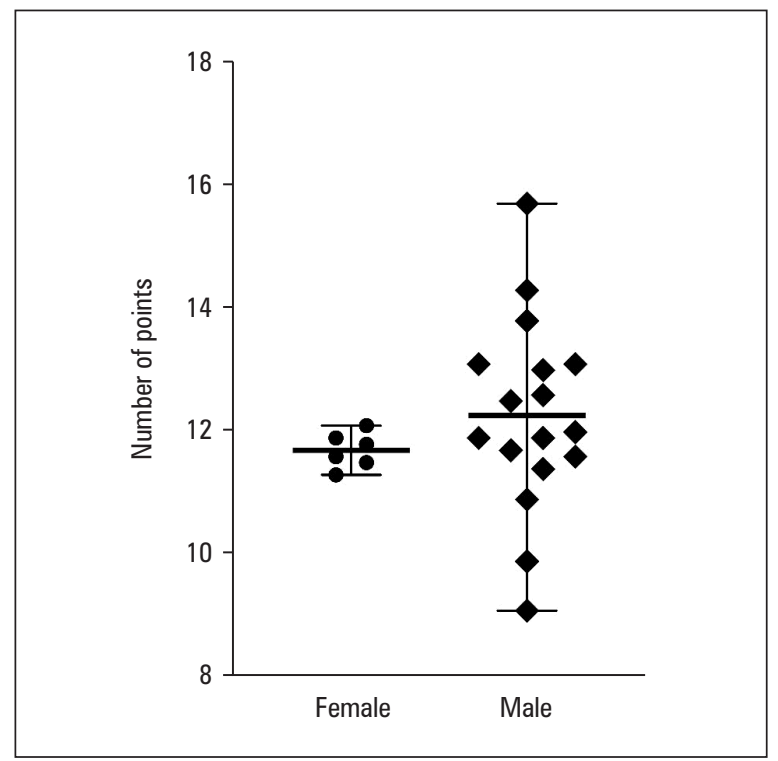

Figure 1. Pulse wave velocity (PWV) values by gender distribution

Results outlined in Table 1 demonstrates a positive correlation between fibrinogen and PWV $(\mathrm{p}=0.04$, Pearson's $r=0.58$ ) and between serum glucose and PWV ( $\mathrm{p}=0.01$, Pearson's $r=0.57$ ).
Pulse wave velocity $(\mathrm{m} / \mathrm{s})$ was analysed by gender distribution as presented in Figure 1; within the two subgroups, no statistically significant difference was identified $(\mathrm{p}=0.46)$.

Age and ABPM BPLab derived data (MAP, PWV) correlated to neutrophil-to-lymphocyte ratio were analysed, as presented in Table 2.

A correlation between PWV and NLR $(p<0.01)$ was found in our statistical analysis. We could not identify a statistically significant correlation between NLR and MAP, although there is a positive correlation $(\mathrm{p}=0.48)$ (see Tab. 2).

In the interest of analysing the impact of disease duration and age-dependent NLR on PWV, the study population was divided according to age ( $\geq 50$ years and $\geq 60$ years). Analysis of Pearson's correlation test regarding the association between age and age-dependent NLR to PWV is presented in Table 3.

Regarding age-dependent NLR, for subjects in age groups 50 years and older, we identified a correlation between NLR and PWV as is illustrated in Table 3 $(\mathrm{p}=0.01$, Pearson's $r=0.71)$. For subjects in the age group 60 years and older, we identified a correlation between NLR and PWV as seen in Table 3 ( $\mathrm{p}=0.01$, Pearson's $r=0.85$ ).

Table 2. Neutrophil-to-lymphocyte (NLR) ratio correlation to 24-hour ambulatory blood pressure monitoring (ABPM) derived data, and age in the studied group

\begin{tabular}{|l|c|c|c|c|c|}
\hline Parameters & & NLR & MAP & PWV & Age \\
\hline \multirow{3}{*}{ NLR } & Pearson's $r$ & 1 & 0.15 & 0.65 & 0.37 \\
\cline { 2 - 6 } & p value & - & 0.48 & 0.00 & 0.07 \\
\hline \multirow{3}{*}{ MAP } & Pearson's $r$ & 0.15 & 1 & 0.51 & -0.02 \\
\cline { 2 - 6 } & p value & 0.48 & - & 0.01 & 0.94 \\
\hline \multirow{3}{*}{ PWV } & Pearson's $r$ & 0.65 & 0.51 & 1 & 0.36 \\
\cline { 2 - 6 } & p value & 0.00 & 0.01 & - & 0.08 \\
\hline \multirow{2}{*}{ Age } & Pearson's $r$ & 0.37 & -0.02 & 0.36 & 1 \\
\cline { 2 - 6 } & p value & 0.07 & 0.94 & 0.08 & - \\
\hline
\end{tabular}

MAP — mean arterial pressure; PWV — pulse wave velocity

Table 3. Pulse wave velocity (PWV) correlation to age and neutrophil-to-lymphocyte (NLR) in studied subjects

\begin{tabular}{|l|c|c|c|c|}
\hline \multirow{2}{*}{ Parameters } & \multicolumn{3}{|c|}{ PWV } \\
\cline { 2 - 5 } & $\mathbf{p}$ & $\mathbf{9 5 \%} \mathbf{C l}$ & Pearson's $\boldsymbol{r}$ & $\mathbf{N}$ \\
\hline Age $\geq 50$ & 0.09 & -0.07 to 0.73 & -0.40 & 18 \\
\hline Age $\geq 60$ & 0.55 & -0.51 to 0.77 & 0.22 & 9 \\
\hline NLR for age $\geq 50$ & 0.01 & 0.36 to 0.88 & 0.71 & 9 \\
\hline NLR for age $\geq 60$ & 0.01 & 0.45 to 0.96 & 0.85 & 18 \\
\hline
\end{tabular}

$\mathrm{Cl}$ - confidence interval; $\mathrm{N}$ - number of patients 


\section{Discussions}

The neutrophil-to-lymphocyte ratio is a simple, readily available, inexpensive marker of inflammation and is expected to be a marker of oxidative stress as well. A relation between NLR and hypertension has been reported in previous studies, as increased NLR in elderly hypertensive patients was associated with increased all-cause mortality [33]. Moreover, NLR was raised in prevalent hypertensive patients and also in individuals with incident hypertension [34, 35].

Even though NLR is a reliable marker of inflammation, it should be considered that NLR levels may be influenced by different situations such as hydration fluctuation [36]. Furthermore, when interpreting different study results concerning NLR values, antihypertensive drugs should be considered, as amlodipine and valsartan administration may decrease NLR [24]. Because use of valsartan reduced interleukin 6 and tumour necrosis factor $\alpha$ in hypertensive patients in previous studies and valsartan and amlodipine as well were reported to decrease oxidative stress and inflammatory response [24], we believe that patients benefiting from these antihypertensive regimens will experience reduced NLR values in comparison with patients treated with other antihypertensive regiments, thus preventing us from properly assessing the correlation between NLR and PWV.

Neutrophil-to-lymphocyte ratio is also an accurate marker for the atherosclerotic disease, which might be related to the influence of the autonomic nervous system as lymphocytes present cholinergic receptors while granulocytes expose adrenergic receptors. Sympathetic nervous system stimulation can increase granulocytes number, and parasympathetic nervous system stimulation can increase lymphocytes [37]. Consequently, vessel sympathetic overactivation, which is encountered in hypertension, heart failure, acute coronary syndromes and arrhythmias, can lead to increased neutrophils and endothelial dysfunction, an essential step in atherosclerotic plaque development [38]. Furthermore, central and peripheral artery stiffness are reported as enhanced under the influence of increased muscle sympathetic nerve activity, which leads to the reasonable assumption that sympathetic hyperactivity might be a contributing factor to the association between increased NLR and PWV [39].

The correlation between NLR and arterial stiffness has been reported in multiple previous studies, particularly in individuals diagnosed with chronic inflammatory conditions. Statistically significant associations were found in patients with systemic lupus erythematosus, a pathology long known to bare important traditional and non-traditional CV risk factors, and also in patients with inflammatory bowel disease, a pathology with a low prevalence of traditional CV risk factors [30, 40].

There are few studies investigating a relation between NLR and PWV that included individuals without a significant inflammatory condition. Additionally, records aiming to identify an association between PWV and NLR in hypertensive patients are limited as well. Nevertheless, there are data describing NLR as a valid indicator for arterial stiffness in hypertensive patients [41].

Our study identified a statistically significant association between NLR and arterial stiffness assessed by PWV analysis in a homogenous group of hypertensive patients. Furthermore, we identified a positive correlation between fibrinogen and PWV as well. Even though a relation between NLR and arterial stiffness in hypertensive patients has been reported before, the novelty of our study consists in the homogenous population included, with controlled hypertension and lacking in confounding factors for NLR. Patients with confounding factors for PWV such as atrial fibrillation, frequent ectopic beats, secondary hypertension, documented or suspected cardiomyopathy were excluded in order to assess a relationship between PWV and NLR accurately. For the same purpose, patients with inflammatory conditions (diabetes, $\mathrm{CKD}$, infectious diseases, malignant tumours, other chronic inflammatory conditions) already known to present increased NLR and a high risk for increased PWV were also excluded.

In patients aged 60 and older, hypertension is the most frequent cardiovascular condition. For proper evaluation of the influence on PWV of disease-duration-dependent hypertension, we further divided our study group according to age as follows: patients $\geq 50$ years and patients $\geq 60$ years. Ageing, which we assume is associated with prolonged hypertensive disease duration, did not have a statistically significant correlation to PWV, most likely due to the small sample group. As expected, aged-dependent NLR was associated with PWV in each age group.

Impaired glucose tolerance stimulates non-enzymatic glycation and cross-linking of collagen, thus contributing to increased arterial stiffness [42]. Glucose serum levels also proved to be correlated to PWV in our study population. We included nondiabetic patients, with incidental low-level hyperglycaemia recorded in a small number of individuals; hence we believe the positive correlation between glycaemia and PWV may be casual in our subjects, mainly as a result of small sample size. 
Our research has several inherent limitations. First, our study was a retrospective observational one which included a cluster of patients from the urban area, highly adherent to antihypertensive treatment, which may have different characteristics from the general hypertensive population. We did not include confounding factors (i.e. regional anthropometric indices) that could influence our results. Second, PWV was not determined prior to BP optimal control. In addition, the sample size of our research work was limited due to recent relative availability to use the BPLab device, which may contribute to the results.

\section{Conclusion}

There is an evident relation between increased neutrophil-to-lymphocyte ratio and increased pulse wave velocity in controlled hypertensive patients with no confirmation of chronic inflammatory conditions. Further directed investigations are required through prospective studies.

\section{Author contributions}

Conceptualization: A.V., C.F.S., and C.F.B.; methods: A.V., C.F.S., D.N.P, and C.F.B.; statistical analysis: A.V., T.Z., and C.F.B.; validation: A.V., T.Z., C.F.S., D.N.P, and C.F.B; investigation: A.V., T.Z., C.F.S., and D.N.P.; resources: A.V., C.F.S., and D.N.P.; data curation: A.V., C.F.S., C.F.B.; writing — original draft preparation: A.V., T.Z., C.F.S., D.N.P., and C.F.B.; writing - review and editing: A.V., T.Z., C.F.S., D.N.P, and C.F.B.; figures: A.V., T.Z., C.F.S., D.N.P., and C.F.B.; supervision: A.V., C.F.S., and C.F.B.; project administration: A.V., and C.F.B. All authors have read and agreed to the published version of the manuscript.

None.

\section{Funding}

\section{Conflicts of interest}

The authors declare no potential conflict of interest regarding this paper.

\section{References}

1. Benjamin E, Virani S, Callaway C, et al. Heart Disease and Stroke Statistics - 2018 Update: A Report From the American Heart Association. Circulation. 2018; 137(12), doi: 10.1161/ cir.0000000000000558, indexed in Pubmed: 29386200.

2. Lee HY, Oh BH. Aging and Arterial Stiffness. Circ J. 2010; 74(11): 2257-2262, doi: 10.1253/circj.cj-10-0910, indexed in Pubmed: 20962429.

3. Virmani R, Kolodgie F, Burke A, et al. Lessons From Sudden Coronary Death. A Comprehensive Morphological Classification
Scheme for Atherosclerotic Lesions. Arterioscler Thromb Vasc Biol. 2000; 20(5): 1262-1275, doi: 10.1161/01.atv.20.5.1262, indexed in Pubmed: 10807742.

4. Cavalcante J, Lima J, Redheuil A, et al. Aortic Stiffness. J Am Coll Cardiol. 2011; 57(14): 1511-1522, doi: 10.1016/j. jacc.2010.12.017, indexed in Pubmed: 21453829.

5. Vlachopoulos C, Aznaouridis K, Stefanadis C. Prediction of Cardiovascular Events and All-Cause Mortality With Arterial Stiffness. J Am Coll Cardiol. 2010; 55(13): 1318-1327, doi: 10.1016/j. jacc.2009.10.061, indexed in Pubmed: 20338492.

6. Omboni S, Posokhov I, Parati G, et al. Ambulatory blood pressure and arterial stiffness web-based telemonitoring in patients at cardiovascular risk. First results of the VASOTENS (Vascular health ASsessment Of The hypertENSive patients) Registry. J Clin Hypertens (Greenwich). 2019; 21(8): 1155-1168, doi: 10.1111/jch.13623, indexed in Pubmed: 31294910.

7. Stehouwer C, Henry R, Ferreira I. Arterial stiffness in diabetes and the metabolic syndrome: a pathway to cardiovascular disease. Diabetologia. 2008; 51(4): 527-539, doi: 10.1007/s00125-007-0918-3, indexed in Pubmed: 18239908.

8. Kinlay S, Creager M, Fukumoto M, et al. Endothelium-Derived Nitric Oxide Regulates Arterial Elasticity in Human Arteries In Vivo. Hypertension. 2001; 38(5): 1049-1053, doi: 10.1161/ hy1101.095329, indexed in Pubmed: 11711496.

9. Kim HL, Kim SH. Pulse Wave Velocity in Atherosclerosis. Front Cardiovasc Med. 2019; 6: 41, doi: 10.3389/fcvm.2019.00041, indexed in Pubmed: 31024934.

10. Ghosh A, Dharmarajan A, Swain P, et al. Impact of Cardiovascular Factors on Pulse Wave Velocity and Total Vascular Resistance in Different Age Group Patients with Cardiovascular Disorders. Curr Aging Sci. 2019; 11(4): 261-268, doi: 10.2174/1874609812666 190226151500 , indexed in Pubmed: 30813882.

11. Avolio A, Butlin M, Liu YY, et al. Regulation of arterial stiffness: Cellular, molecular and neurogenic mechanisms. Artery Res 2011; 5(4):122-127. DOI: 10 1016/j artres. 2011; 10: 002, doi: 10.1016/j.artres.2011.10.002

12. Townsend R. Arterial stiffness and chronic kidney disease. Curr Opin Nephrol Hypertens. 2015; 24(1): 47-53, doi: $10.1097 /$ mnh.0000000000000086, indexed in Pubmed: 2547001.

13. Williams B, Mancia G, Spiering W, et al. 2018 ESC/ESH Guidelines for the management of arterial hypertension. J Hypertens. 2018; 36(10): 1953-2041, doi: 10.1097/hjh.0000000000001940, indexed in Pubmed: 30234752.

14. Laurent S, Cockcroft J, Bortel LV, et al. Expert consensus document on arterial stiffness: methodological issues and clinical applications. Eur Heart J. 2006; 27(21): 2588-2605, doi: 10.1093/eurheartj/ ehl254, indexed in Pubmed: 17000623.

15. Napoli C, Nigris Fde, Williams-Ignarro S, et al. Nitric oxide and atherosclerosis: An update. Nitric Oxide. 2006; 15(4): 265-279, doi: 10.1016/j.niox.2006.03.011, indexed in Pubmed: 16684613.

16. Wang M, Jiang L, Monticone R, et al. Proinflammation: the key to arterial aging. Trends Endocrinol Metab . 2014; 25(2): 72-79, doi: 10.1016/j.tem.2013.10.002, indexed in Pubmed: 24365513.

17. Thijssen D, Carter S, Green D. Arterial structure and function in vascular ageing: are you as old as your arteries? J Physiol. 2015; 594(8): 2275-2284, doi: 10.1113/jp270597, indexed in Pubmed: 26140618.

18. Bortel LV, Laurent S, Boutouyrie P, et al. Expert consensus document on the measurement of aortic stiffness in daily practice using carotid-femoral pulse wave velocity. J Hypertens. 2012; 30(3): 445-448, doi: 10.1097/hjh.0b013e32834fa8b0, indexed in Pubmed: 22278144.

19. Luzardo L, Lujambio I, Sottolano M, et al. 24-h ambulatory recording of aortic pulse wave velocity and central systolic augmentation: a feasibility study. Hypertens Res. 2012; 35(10): 980-987, doi: 10.1038/hr.2012.78, indexed in Pubmed: 22622282.

20. Horváth I, Németh Á, Lenkey Z, et al. Invasive validation of a new oscillometric device (Arteriograph) for measuring augmentation index, central blood pressure and aortic pulse wave velocity. J Hypertens. 2010; 28(10): 2068-2075, doi: 10.1097/hjh.0b013e32833c8a1a, indexed in Pubmed: 20651604. 
21. Omboni S, Posokhov I, Rogoza A. Evaluation of 24-Hour Arterial Stiffness Indices and Central Hemodynamics in Healthy Normotensive Subjects versus Treated or Untreated Hypertensive Patients: A Feasibility Study. Int J Hypertens. 2015; 2015: 1-10, doi: 10.1155/2015/601812, indexed in Pubmed: 25692032.

22. Mehmet GK. [Inflammation and coronary artery disease: as a new biomarker neutrophil/lymphocyte ratio]. Turk Kardiyol Dern Ars. 2013; 41(3): 191-192, doi: 10.5543/tkda.2013.84484, indexed in Pubmed: 23703552.

23. Balta S, Celik T, Mikhailidis D, et al. The Relation Between Atherosclerosis and the Neutrophil-Lymphocyte Ratio. Clin Appl Thromb Hemost. 2015; 22(5): 405-411, doi: 10.1177/1076029615569568, indexed in Pubmed: 25667237.

24. Karaman M, Balta S, AY S, et al. The Comparative Effects of Valsartan and Amlodipine on vWf Levels and N/L Ratio in Patients with Newly Diagnosed Hypertension. Clin Exp Hypertens. 2013; 35(7): 516-522, doi: 10.3109/10641963.2012.758734, indexed in Pubmed: 23289969.

25. Sharma K, Patel A, Shah K, et al. Is Neutrophil-to-Lymphocyte Ratio a Predictor of Coronary Artery Disease in Western Indians? Int J Inflam. 2017; 2017: 1-8, doi: 10.1155/2017/4136126, indexed in Pubmed: 28811954.

26. Li Y, Chen X, Huang L, et al. Association between neutrophil-lymphocyte ratio and arterial stiffness in patients with acute coronary syndrome. Biosci Rep. 2019; 39(5), doi: 10.1042/bsr20190015, indexed in Pubmed: 30992389.

27. Forget P, Khalifa C, Defour JP, et al. What is the normal value of the neutrophil-to-lymphocyte ratio? BMC Res Notes. 2017; 10(1): 12, doi: 10.1186/s13104-016-2335-5, indexed in Pubmed: 28057051.

28. Park BJ, Shim JY, Lee HR, et al. Relationship of neutrophil-lymphocyte ratio with arterial stiffness and coronary calcium score. Clin Chim Acta. 2011; 412(11-12): 925-929, doi: 10.1016/j. cca.2011.01.021, indexed in Pubmed: 21266168.

29. Yang XF, Ding FM, Ye YC, et al. Relationship between Neutrophilto-Lymphocyte Ratio and Pulse Wave Velocity in Young Patients with Systemic Lupus Erythematosus. Chin Med J (Engl). 2018; 131(1): 10-15, doi: 10.4103/0366-6999.221272, indexed in Pubmed: 29271374.

30. Zanoli L, Ozturk K, Cappello M, et al. Inflammation and Aortic Pulse Wave Velocity: A Multicenter Longitudinal Study in Patients With Inflammatory Bowel Disease. J Am Heart Assoc. 2019; 8(3): e010942, doi: 10.1161/jaha.118.010942, indexed in Pubmed: 30712441.

31. Wang H, Hu Y, Geng Y, et al. The relationship between neutrophil to lymphocyte ratio and artery stiffness in subtypes of hypertension. J Clin Hypertens (Greenwich). 2017; 19(8): 780-785, doi: 10.1111/ jch.13002, indexed in Pubmed: 28480636.
32. Omboni S, Posokhov I, Kotovskaya Y, et al. Twenty-Four-Hour Ambulatory Pulse Wave Analysis in Hypertension Management: Current Evidence and Perspectives. Curr Hypertens Rep. 2016; 18(10), doi: 10.1007/s1 1906-016-0681-2, indexed in Pubmed: 27659178.

33. Sun X, Luo L, Zhao X, et al. The neutrophil-to-lymphocyte ratio on admission is a good predictor for all-cause mortality in hypertensive patients over 80 years of age. BMC Cardiovascular Disord. 2017; 17(1), doi: 10.1186/s12872-017-0595-1, indexed in Pubmed: 28646855.

34. Liu X, Zhang Q, Wu H, et al. Blood Neutrophil to Lymphocyte Ratio as a Predictor of Hypertension. Am J Hypertens. 2015; 28(11): 1339-1346, doi: 10.1093/ajh/hpv034, indexed in Pubmed: 25824450.

35. Jhuang YH, Kao TW, Peng TC, et al. Neutrophil to lymphocyte ratio as predictor for incident hypertension: a 9-year cohort study in Taiwan. Hypertens Res. 2019; 42(8): 1209-1214, doi: 10.1038/ s41440-019-0245-3, indexed in Pubmed: 30850753.

36. Balta S, Ozturk C, Yildirim A, et al. The Relation Between Neutrophil-Lymphocyte Ratio and Hypertension. Am J Hypertens. 2015; 28(11): 1386-1386, doi: 10.1093/ajh/hpv137, indexed in Pubmed: 26447180.

37. Abo T, Kawamura T. Immunomodulation by the Autonomic Nervous System: Therapeutic Approach for Cancer, Collagen Diseases, and Inflammatory Bowel Diseases. Ther Apher. 2002; 6(5): 348-357, doi: 10.1046/j.1526-0968.2002.00452.x, indexed in Pubmed: 12423529

38. Manolis AJ, Poulimenos LE, Kallistratos MS, et al. Sympathetic overactivity in hypertension and cardiovascular disease. . Curr Vasc Pharmacol. 2014; 12(1): 4-15, doi: 10.2174/157016111131199 90140, indexed in Pubmed: 23905597.

39. Świerblewska E, Hering D, Kara T, et al. An independent relationship between muscle sympathetic nerve activity and pulse wave velocity in normal humans. J Hypertens. 2010; 28(5): 979-984, doi: $10.1097 /$ hih.0b013e328336ed9a, indexed in Pubmed: 20408258.

40. Yang XF, Ding FM, Ye YC, et al. Relationship between Neutrophilto-Lymphocyte Ratio and Pulse Wave Velocity in Young Patients with Systemic Lupus Erythematosus. Chin Med J (Engl). 2018; 131(1): 10-15, doi: 10.4103/0366-6999.221272, indexed in Pubmed: 29271374.

41. Wang H, Hu Y, Geng Y, et al. The relationship between neutrophil to lymphocyte ratio and artery stiffness in subtypes of hypertension. J Clin Hypertens (Greenwich). 2017; 19(8): 780-785, doi: 10.1111/ jch.13002, indexed in Pubmed: 28480636.

42. Flier J, Underhill L, Brownlee M, et al. Advanced Glycosylation End Products in Tissue and the Biochemical Basis of Diabetic Complications. N Engl J Med. 1988; 318(20): 1315-1321, doi: 10.1056/ nejm198805193182007, indexed in Pubmed: 3283558. 